\title{
EQUALITY OF ESSENTIAL SPECTRA OF CERTAIN QUASISIMILAR SEMINORMAL OPERATORS
}

\author{
L. R. WILLIAMS
}

\begin{abstract}
Let $A$ and $B$ be quasisimilar seminormal operators on a separable, infinite dimensional complex Hilbert space. Several conditions which imply that $A$ and $B$ have equal essential spectra are presented. For example, if $A$ and $B$ are both biquasitriangular then $A$ and $B$ have equal essential spectra.
\end{abstract}

Let $\mathcal{H}$ denote a separable, infinite dimensional complex Hilbert space, and let $\mathcal{L}(\mathcal{H})$ denote the algebra of all bounded linear operators on $\mathcal{H}$. (We shall use the term operator to mean an element of $\mathcal{L}(\mathcal{H})$. Unless specified otherwise, all operators are assumed to be acting on the Hilbert space $\mathcal{H}$.) An operator $T$ is said to be hyponormal if $T T^{*} \leqslant T^{*} T$. If $T$ is an operator and $T^{*}$ is hyponormal, then $T$ is said to be cohyponormal. If $T$ is either hyponormal or cohyponormal, then $T$ is called a seminormal operator. An operator $X$ having trivial kernel and dense range is called a quasiaffinity. Operators $A$ and $B$ are said to be quasisimilar if there exist quasiaffinities $X$ and $Y$ such that $X A=B X$ and $A Y=Y B$. If $T$ belongs to $\mathcal{L}(\mathcal{H})$, we shall let $\mathscr{K}(T)$ denote the kernel of $T, \sigma(T)$ the spectrum of $T$, and $\sigma_{e}(T)$ the essential spectrum of $T$, i.e., the spectrum of $\pi(T)$, where $\pi$ is the natural quotient map of $\mathcal{L}(\mathcal{H})$ onto the Calkin algebra $\mathscr{L}(\mathcal{H}) / \mathcal{C}(\mathcal{C}$ denotes the norm-closed ideal of all compact operators in $\mathcal{E}(\mathcal{H})$ ).

It is known that quasisimilar normal operators are unitarily equivalent (cf. [3]). Thus quasisimilar normal operators have equal spectra and essential spectra. S. Clary constructed an example in [2] which shows that quasisimilar hyponormal operators need not be similar. Nevertheless, he proved that quasisimilar hyponormal operators do have equal spectra. Clary's proof, with only modest modifications, shows that quasisimilar seminormal operators have equal spectra. In view of the above results, it is natural to pose the following question: Do quasisimilar seminormal operators have equal essential spectra? At the present we are unable to answer this question. However, in this note, we do show that the answer is "yes" in several interesting cases.

Let $A$ and $B$ be operators and $X$ a quasiaffinity satisfying $X A=B X$. M. Radjabalipour proved recently in [10] that if $A^{*}$ and $B$ are hyponormal, then $A$ and $B$ are unitarily equivalent normal operators. (H. Radjavi and P. Rosenthal proved earlier in [11] that the above result is valid in the subnormal case.) Let $A$ and $B$ be quasisimilar seminormal operators. In view of Radjabalipour's result, in order to

Presented to the Society, January 24, 1979; received by the editors December 12, 1978.

AMS (MOS) subject classifications (1970). Primary 47B20; Secondary 47A10

Key words and phrases. Hyponormal operator, cohyponormal operator, seminormal operator, quasiaffinity, quasisimilar, essential spectrum. 0002-9939/80/0000-0060/\$02.75 
determine whether or not $\sigma_{e}(A)=\sigma_{e}(B)$, it suffices to assume that both $A$ and $B$ are hyponormal. Therefore, in this note, we shall consider the hyponormal case only. (We acknowledge that the question of whether quasisimilar hyponormal operators have equal essential spectra was originally raised by Clary in [2].) We remark that the problem under study appears to be open even in the case of subnormal operators.

For ease of reference, we state without proof the following lemma which is due to Clary [2].

Lemma 1. Suppose that $A, B$, and $X$ are operators where $B$ is hyponormal, $X$ has dense range, and $X A=B X$. If $A$ is invertible, then $B$ is also invertible.

Recall that an operator $T$ is said to be a Fredholm operator if both $\mathscr{K}(T)$ and $\mathscr{K}\left(T^{*}\right)$ are finite dimensional and the range of $T$ is closed. For $T$ in $\mathcal{L}(\mathcal{H})$ it is well known that $\sigma_{e}(T)=\{\lambda \in C: T-\lambda$ is not Fredholm $\}$. If $T$ is a Fredholm operator, let $i(T)=\operatorname{dim} \mathcal{K}(T)-\operatorname{dim} \mathscr{K}\left(T^{*}\right)$ denote the index of $T$.

Observe that if $T$ is hyponormal in $\mathcal{L}(\mathcal{H})$, then $\left\|T^{*} x\right\| \leqslant\|T x\|$ for each $x$ in $\mathcal{H}$. Thus $\mathscr{K}(T) \subseteq \mathscr{K}\left(T^{*}\right)$. Hence $\mathcal{K}(T)$ reduces $T$.

Lemma 2. Suppose that $A$ and $B$ are quasisimilar hyponormal operators. Then $A$ is a Fredholm operator satisfying $i(A)=0$ if and only if $B$ is a Fredholm operator satisfying $i(B)=0$.

Proof. There exist quasiaffinities $X$ and $Y$ such that $X A=B X$ and $A Y=Y B$. Now suppose that $A$ is a Fredholm operator satisfying $i(A)=0$. Since $A$ and $B$ are quasisimilar, it follows that $\operatorname{dim} \mathscr{K}(A)=\operatorname{dim} \mathscr{K}(B)$ and $\operatorname{dim}\left(\mathcal{K}(A)^{\perp}\right)=$ $\operatorname{dim}\left(\mathcal{K}(B)^{\perp}\right)$. Thus, without loss of generality, we may assume that $\mathscr{K}(A)=\mathscr{K}(B)$. (Replace $B$ by $U^{*} B U, X$ by $U^{*} X$, and $Y$ by $Y U$ for a suitable unitary operator $U$.) Since $A$ is a hyponormal Fredholm operator satisfying $i(A)=0$, we have $\mathscr{K}(A)=$ $\mathcal{K}\left(A^{*}\right)$. Note that $\mathcal{K}(A)$ is an invariant subspace for the operator $X$. The matrices of $A, B$, and $X$ with respect to the decomposition $\mathcal{K}=\mathscr{K}(A)^{\perp} \oplus \mathscr{K}(A)$ are

$$
\left[\begin{array}{cc}
A_{1} & 0 \\
0 & 0
\end{array}\right], \quad\left[\begin{array}{cc}
B_{1} & 0 \\
0 & 0
\end{array}\right], \quad \text { and }\left[\begin{array}{cc}
X_{1} & 0 \\
X_{2} & X_{3}
\end{array}\right] \text {, }
$$

respectively, where $A_{1}$ is invertible, $B_{1}$ is hyponormal, and $X_{1}$ has dense range in $\mathcal{K}(A)^{\perp}$. The equation $X A=B X$ implies that $X_{1} A_{1}=B_{1} X_{1}$. Hence, by Lemma 1 , $B_{1}$ is also invertible. Thus $B$ is a Fredholm operator satisfying $i(B)=0$. Hence, by symmetry, the proof is complete.

An operator $T$ in $\mathcal{L}(\mathcal{H})$ is said to be quasitriangular if there exists a sequence $\left\{P_{n}\right\}$ of projections of finite rank such that $P_{n} \rightarrow 1_{\mathcal{X}}$ strongly and $\left\|T P_{n}-P_{n} T P_{n}\right\|$ $\rightarrow 0$. An operator $T$ is said to be biquasitriangular if both $T$ and $T^{*}$ are quasitriangular. If $T$ belongs to $\mathcal{L}(\mathcal{H})$, then $T$ is said to be a semi-Fredholm operator if either $\mathscr{K}(T)$ or $\mathscr{K}\left(T^{*}\right)$ is finite dimensional and the range of $T$ is closed. Clearly every Fredholm operator is semi-Fredholm. If $T$ is semi-Fredholm, then, as in the case of a Fredholm operator, let $i(T)=\operatorname{dim} \mathcal{K}(T)-\operatorname{dim} \mathcal{K}\left(T^{*}\right)$ denote the index of $T$. The class of biquasitriangular operators can be characterized as follows: $T$ is 
biquasitriangular if and only if for each complex number $\lambda$ such that $T-\lambda$ is semi-Fredholm, $i(T-\lambda)=0$ (cf. [1], [4], [5]).

In the case of biquasitriangular hyponormal operators, quasisimilarity does imply equality of their essential spectra.

Theorem 1. Suppose that $A$ and $B$ are quasisimilar hyponormal operators. If $A$ and $B$ are also biquasitriangular, then $\sigma_{e}(A)=\sigma_{e}(B)$.

Proof. Suppose that $\lambda \notin \sigma_{e}(A)$. Then $A-\lambda$ is a Fredholm operator and, since $A$ is biquasitriangular, $i(A-\lambda)=0$. Also $A-\lambda$ and $B-\lambda$ are quasisimilar hyponormal operators. Thus, by Lemma $2, B-\lambda$ is also a Fredholm operator, which implies that $\lambda \notin \sigma_{e}(B)$. We have shown that $\sigma_{e}(B) \subseteq \sigma_{e}(A)$. By symmetry, $\sigma_{e}(A) \subseteq$ $\sigma_{e}(B)$. Therefore, $\sigma_{e}(A)=\sigma_{e}(B)$.

Theorem 2. Suppose that $A$ and $B$ are hyponormal operators and there exist quasiaffinities $X$ and $Y$ such that $X A=B X$ and $A Y=Y B$. If either $X$ or $Y$ is compact, then $\sigma_{e}(A)=\sigma_{e}(B)$.

Proof. Without loss of generality assume that $A$ and $B$ are nonzero. Suppose that $X$ or $Y$ is compact. Then the operator $Y X$ is a compact quasiaffinity that commutes with $A$. Hence, according to Theorem 5.1 of [7], if $\lambda$ is a complex number such that $A-\lambda$ is a Fredholm operator, then $i(A-\lambda)=0$. This fact along with Lemma 2 allows us to conclude that $\sigma_{e}(B) \subseteq \sigma_{e}(A)$. By symmetry, the proof is complete.

In regard to Theorem 1, we shall show in Example 2 that quasisimilar biquasitriangular hyponormal operators need not be similar. In regard to Theorem 2, we ask the following question: If $A$ and $B$ are operators satisfying the hypotheses of Theorem 2 , then are $A$ and $B$ necessarily unitarily equivalent or similar?

In the following theorems, we present some classes of hyponormal operators for which quasisimilarity of two operators in a class implies equality of their essential spectra. We shall need the following lemma.

LEMMA 3. If $A$ and $B$ are quasisimilar hyponormal operators and $\mathcal{K}(A)=\mathscr{K}(B)$, then $A_{1}=A \mid \mathscr{K}(A)^{\perp}$ and $B_{1}=B \mid \mathscr{K}(B)^{\perp}$ are quasisimilar hyponormal operators.

Proof. There exist quasiaffinities $X$ and $Y$ such that $X A=B X$ and $A Y=Y B$. The subspace $\mathscr{K}(A)$ is invariant under both $X$ and $Y$ Thus the matrices of $A, B, X$, and $Y$ with respect to the decomposition $\mathcal{K}=\mathscr{K}(A)^{\perp} \oplus \mathscr{K}(A)$ are

$$
\left[\begin{array}{cc}
A_{1} & 0 \\
0 & 0
\end{array}\right], \quad\left[\begin{array}{cc}
B_{1} & 0 \\
0 & 0
\end{array}\right], \quad\left[\begin{array}{cc}
X_{1} & 0 \\
X_{2} & X_{3}
\end{array}\right], \quad \text { and }\left[\begin{array}{cc}
Y_{1} & 0 \\
Y_{2} & Y_{3}
\end{array}\right] \text {, }
$$

respectively. It is easy to verify that the ranges of $X_{1}$ and $Y_{1}$ are dense in $\mathscr{K}(A)^{\perp}$. We now show that $\mathscr{K}\left(X_{1}\right)=\mathcal{K}\left(Y_{1}\right)=\{0\}$. Suppose that $z \in \mathscr{K}\left(X_{1}\right)$. Then $B X(z$ $\oplus 0)=0$. The equation $X A=B X$ implies that $z \in \mathscr{K}\left(A_{1}\right)$. This implies that $z=0$; thus $\mathcal{K}\left(X_{1}\right)=\{0\}$. Likewise, $\mathscr{K}\left(Y_{1}\right)=\{0\}$. So $X_{1}$ and $Y_{1}$ are quasiaffinites on $\mathscr{K}(A)^{\perp}$ and the equations $X A=B X$ and $A Y=Y B$ imply that $X_{1} A_{1}=B_{1} X_{1}$ and $A_{1} Y_{1}=Y_{1} B_{1}$. Hence $A_{1}$ and $B_{1}$ are quasisimilar. The operators $A_{1}$ and $B_{1}$ are clearly hyponormal. 
REMARK. If we allow quasiaffinites to be bounded linear transformations between two Hilbert spaces rather than operators on a given Hilbert space, then the condition $\mathscr{K}(A)=\mathscr{K}(B)$ is unnecessary in Lemma 3 . However, in the proofs that follow, if $A$ is quasisimilar to $B$, then there is no loss of generality in assuming that $\mathscr{K}(A)=\mathscr{K}(B)$. (See the proof of Lemma 2.)

A. Lambert proved in [9] that two quasisimilar injective unilateral weighted shifts are similar. The following theorem shows that the same is true for quasisimilar hyponormal (possibly noninjective) unilateral weighted shifts.

THeOREM 3. If $A$ and $B$ are hyponormal unilateral weighted shifts and $A$ is quasisimilar to $B$, then $A$ is similar to $B$.

Proof. We assume that $\mathscr{K}(A)=\mathscr{K}(B)$. Since $A$ and $B$ are hyponormal unilateral weighted shifts, it follows that $A_{1}=A \mid \mathcal{K}(A)^{\perp}$ and $B_{1}=B \mid \mathcal{K}(A)^{\perp}$ are injective unilateral weighted shifts. Lemma 3 implies that $A_{1}$ is quasisimilar to $B_{1}$. Hence, by Lambert's result [9], $A_{1}$ is similar to $B_{1}$. Therefore, $A$ and $B$ are similar.

COROLlaRY 1. If $A$ and $B$ are hyponormal unilateral weighted shifts and $A$ is quasisimilar to $B$, then $\sigma_{e}(A)=\sigma_{e}(B)$.

LEMMA 4. If $A$ and $B$ are hyponormal injective bilateral weighted shifts and $A$ is quasisimilar to $B$, then $\sigma_{e}(A)=\sigma_{e}(B)$.

Proof. L. A. Fialkow showed in [6] that if either $A$ or $B$ is invertible, then $A$ is similar to $B$, in which case $\sigma_{e}(A)=\sigma_{e}(B)$. So we may assume that neither $A$ nor $B$ is invertible. It follows from Ciary's result [2] that $\sigma(A)=\sigma(B)$. (Fialkow showed also in [6] that any two quasisimilar injective bilateral weighted shifts have equal spectra.) Since hyponormal operators are spectraloid, we have $\|A\|=\|B\|$. We now proceed to describe $\sigma_{e}(A)$ and $\sigma_{e}(B)$. Since $A$ is an injective bilateral weighted shift on $\mathcal{H}$, there exist a sequence $\left\{\alpha_{n}\right\}_{n=-\infty}^{\infty}$ of nonzero complex numbers and an orthonormal basis $\left\{e_{n}\right\}_{n=-\infty}^{\infty}$ for $\mathcal{H}$ such that $A e_{n}=\alpha_{n} e_{n+1}, \quad n$ $=\ldots-2,-1,0,1,2, \ldots$. Since $A$ is hyponormal, we have $\left|\alpha_{n}\right| \leqslant\left|\alpha_{n+1}\right|$, for each integer $n$. Since $A$ is also not invertible, $\lim _{n \rightarrow-\infty}\left|\alpha_{n}\right|=0$. Note also that $\lim _{n \rightarrow \infty}\left|\alpha_{n}\right|$ $=\|A\|$. It follows that there exists a compact weighted shift $K$ such that $(A+K) e_{n}$ $=\gamma_{n} e_{n+1}$, where $\gamma_{n}=0$ for $n \leqslant 0$ and $\gamma_{n}=\|A\|$ for $n>0$. Thus $A+K$ is unitarily equivalent to $0_{\mathscr{C}} \oplus\|A\| S$, where $S$ is the unilateral shift. Thus $\sigma_{e}(A)=$ $\{0\} \cup\{\lambda \in C:|\lambda|=\|A\|\}$. The same argument shows that $\sigma_{e}(B)=\{0\} \cup\{\lambda \in$ $C:|\lambda|=\|B\|\}$. Therefore, $\sigma_{e}(A)=\sigma_{e}(B)$ since $\|A\|=\|B\|$.

In view of Lemma 4 , the proof of the following theorem is similar to that of Theorem 3.

THEOREM 4. If $A$ and $B$ are hyponormal (possibly noninjective) bilateral weighted shifts and $A$ is quasisimilar to $B$, then $\sigma_{e}(A)=\sigma_{e}(B)$.

The following example shows that quasisimilar hyponormal bilateral weighted shifts need not be similar.

EXAMPLE 1. Let $\alpha_{n}=1 / 2^{2 n}$ for $n \geqslant 0$ and $\alpha_{n}=1$ for $n<0$; let $\beta_{n}=1 / 2^{2 n-1}$ for $n \geqslant 0$ and $\beta_{n}=1$ for $n<0$. Let $W_{\alpha}$ and $W_{\beta}$ be the bilateral weighted shifts with 
weight sequences $\left\{\alpha_{n}\right\}_{n=-\infty}^{\infty}$ and $\left\{\beta_{n}\right\}_{n=-\infty}^{\infty}$, respectively. L. A. Fialkow shows in [6] that $W_{\alpha}$ and $W_{\beta}$ are quasisimilar but not similar. Note that $W_{\alpha}$ is cohyponormal. Let $\beta_{n}^{\prime}=\beta_{n}$ for $n \neq 0$ and $\beta_{0}^{\prime}=1$. The bilateral weighted shift $W_{\beta^{\prime}}$ having weight sequence $\left\{\beta_{n}^{\prime}\right\}_{n=-\infty}^{\infty}$ is cohyponormal and similar to $W_{\beta}$. Thus $W_{\alpha}^{*}$ and $W_{\beta^{\prime}}^{*}$ are quasisimilar hyponormal bilateral weighted shifts that are not similar.

An important class of hyponormal operators is the class of isometries. T. B. Hoover proved in [8] that two quasisimilar isometries are unitarily equivalent; thus they have equal spectra and essential spectra. The following theorem extends this result to the class of hyponormal partial isometries.

THEOREM 5. If $A$ and $B$ are hyponormal partial isometries and $A$ is quasisimilar to $B$, then $A$ is unitarily equivalent to $B$.

Proof. We assume that $\mathscr{K}(A)=\mathcal{K}(B)$. Using Lemma 3, we conclude that the operators $A_{1}=A \mid \mathcal{K}(A)^{\perp}$ and $B_{1}=B \mid \mathscr{K}(A)^{\perp}$ are quasisimilar isometries. So, by Hoover's result [8], $A_{1}$ and $B_{1}$ are unitarily equivalent. Thus $A$ and $B$ are unitarily equivalent.

COROllary 2. If $A$ and $B$ are hyponormal partial isometries and $A$ is quasisimilar to $B$, then $\sigma_{e}(A)=\sigma_{e}(B)$.

Let $A$ be cohyponormal and $B$ be a nonnormal hyponormal operator. It follows from Radjabalipour's result [10] that there exists no quasiaffinity $X$ such that $X A=B X$. Moreover, J. G. Stampfli and B. L. Wadhwa have proved that if $B$ is the unilateral shift, then there exists no nonzero operator $X$ such that $X A=B X$ (cf. [14, Theorem 3]). The following theorem extends this result of Stampfli and Wadhwa.

THEOREM 6. Suppose that $A$ is cohyponormal and that $B$ is a nonnormal hyponormal injective weighted shift (unilateral or bilateral). Then there exists no nonzero operator $X$ such that $X A=B X$.

Proof. The proof is a modification of that of Theorem 3 of [14]. For definiteness, let $B$ be a nonnormal hyponormal injective bilateral shift with weight sequence $\left\{\alpha_{n}\right\}_{n=-\infty}^{\infty}$ with respect to an orthonormal basis $\left\{e_{n}\right\}_{n=-\infty}^{\infty}$ for $\mathcal{H}$. (The proof in the case of the unilateral shift is similar.) Suppose that $X A=B X$, where $A$ is cohyponormal and $X$ is an operator. Since $\left|\alpha_{n}\right| \leqslant\left|\alpha_{n+1}\right|$ for each integer $n$, we have $\lim _{n \rightarrow+\infty}\left|\alpha_{n}\right|=\|B\|$. Let $r=\lim _{n \rightarrow-\infty}\left|\alpha_{n}\right|$; and let $\Lambda=\{\lambda \in C: r<|\lambda|<$ $\|B\|\}$. (Note that $r<\|B\|$ since $B$ is nonnormal.) Let $\delta_{n}=\left(\alpha_{0} \cdot \alpha_{1} \cdots \cdot \alpha_{n-1}\right)^{-1}$ for $n>0, \delta_{-n}=\alpha_{-1} \cdot \alpha_{-2} \cdots \cdot \alpha_{-n}$ for $n>0$, and $\delta_{0}=1$. It is easy to verify that $\sum_{n=-\infty}^{\infty}\left|\delta_{n} \lambda^{n}\right|^{2}$ converges for each $\lambda$ in $\Lambda$. For $\lambda$ in $\Lambda$, let $f_{\lambda}=\sum_{n=-\infty}^{\infty} \bar{\delta}_{n} \lambda^{n} e_{n}$. Then $B^{*} f_{\lambda}=\lambda f_{\lambda}$ for each $\lambda$ in $\Lambda$. (Actually $\Lambda$ is the point spectrum of $B^{*}$. See [12] and [13] for a discussion of the spectra of weighted shift operators.) Rewriting our equation as $A^{*} X^{*}=X^{*} B^{*}$, we get $A^{*}\left(X^{*} f_{\lambda}\right)=\lambda X^{*} f_{\lambda}$. Since $A^{*}$ is hyponormal, $X^{*} f_{\lambda}$ is orthogonal to $X^{*} f_{\mu}$ for $\lambda \neq \mu$. Since $\mathcal{H}$ is separable, we have $X^{*} f_{\lambda}=0$ for all but countably many $\lambda$ 's in $\Lambda$. Since the map $\lambda \rightarrow f_{\lambda}$ from $\Lambda$ into $\mathcal{H}$ (norm-topology) is continuous, it follows that $X^{*} f_{\lambda}=0$ for each $\lambda$ in $\Lambda$. We next show that the 
linear span of $\left\{f_{\lambda}\right\}_{\lambda \in \Lambda}$ is dense in $\mathcal{H}$. Suppose that $g=\sum_{n=-\infty}^{\infty} \beta_{n} e_{n}$ is a vector in $\mathcal{H}$ such that $\left(f_{\lambda}, g\right)=0$ for each $\lambda$ in $\Lambda$. The series $\sum_{n=-\infty}^{\infty} \bar{\beta}_{n} \bar{\delta}_{n} \lambda^{n}=\left(f_{\lambda}, g\right)$ is a Laurent series that converges on $\Lambda$, and thus represents the zero analytic function there. By the uniqueness of the Laurent series, we have $\bar{\beta}_{n} \bar{\delta}_{n}=0$ for each integer $n$. This implies that $\beta_{n}=0$ for each integer $n$; thus $g=0$. Hence the linear span of $\left\{f_{\lambda}\right\}_{\lambda \in \Lambda}$ is dense in $\mathcal{H}$. Therefore $X=0$.

The next example shows that quasisimilar biquasitriangular hyponormal operators need not be similar.

EXAMPLE 2. In Example 1, we constructed two quasisimilar hyponormal bilateral weighted shifts $W_{\alpha}$ and $W_{\beta}$ that are not similar. Close scrutiny of Example 1 will reveal that $W_{\alpha}$ and $W_{\beta}$ are both injective and nonnormal and that $\sigma\left(W_{\alpha}\right)=\sigma\left(W_{\beta}\right)$ $=\{\lambda \in C:|\lambda| \leqslant 1\}$. Let $N$ be a normal operator such that $\sigma(N)=\sigma_{e}(N)=\{\lambda \in$ $C:|\lambda| \leqslant 1\}$. Let $A=W_{\alpha} \oplus N$ and $B=W_{\beta} \oplus N$. It is clear that $A$ and $B$ are quasisimilar hyponormal operators on $\mathcal{H} \oplus \mathscr{H}$. Suppose that $A-\lambda$ is semi-Fredholm. Then $N-\lambda$ is semi-Fredholm, and hence Fredholm. Therefore, $\lambda \in C \backslash$ $\sigma_{e}(N)=C \backslash \sigma(A)$, and thus $i(A-\lambda)=0$. It follows from the spectral characterization of the biquasitriangular operators that $A$ is biquasitriangular. Likewise $B$ is biquasitriangular. We show next that $A$ is not similar to $B$. Suppose, to the contrary, that there exists an invertible operator $C$ such that $C A=B C$. Let

$$
\left[\begin{array}{ll}
Q & R \\
S & T
\end{array}\right] \text { and }\left[\begin{array}{ll}
V & X \\
Y & Z
\end{array}\right]
$$

be the matrices of $C$ and $C^{-1}$, respectively, on $\mathcal{H} \oplus \mathcal{H}$. The equation $C A=B C$ implies that $W_{\beta} R=R N$ and $Q W_{\alpha}=W_{\beta} Q$. The equation $A C^{-1}=C^{-1} B$ implies that $W_{\alpha} X=X N$. Theorem 6 implies that $R=X=0$. Hence the equations $C C^{-1}$ $=C^{-1} C=1_{\mathcal{C} \oplus \mathcal{X}}$ imply that $Q V=V Q=1_{\mathscr{K}}$. Thus we have $W_{\alpha}$ is similar to $W_{\beta}$, which is a contradiction. Therefore, $A$ is not similar to $B$.

REMARK. Observe that the classes of hyponormal operators mentioned in Theorems 3, 4 and 5 include operators that are not biquasitriangular.

Acknowledgment. The author is grateful to the referee for many helpful suggestions.

\section{BIBLIOGRAPHY}

1. C. Apostol, C. Foias and D. Voiculescu, Some results on non-quasitriangular operators. IV, Rev. Roumaine Math. Pures Appl. 18 (1973), 487-514.

2. S. Clary, Equality of spectra of quasisimilar hyponormal operators, Proc. Amer. Math. Soc. 53 (1975), 88-90.

3. R. G. Douglas, On the operator equation $S^{*} X T=X$ and related topics, Acta Sci. Math. (Szeged) 30 (1969), 19-32.

4. R. G. Douglas and C. Pearcy, Invariant subspaces of non-quasitriangular operators, Proc. Conf. on Operator Theory, Lecture Notes in Math., no. 345, Springer-Verlag, Berlin and New York, 1973, pp. 13-57.

5. _ A note on quasitriangular operators, Duke Math. J. 37 (1970), 177-188.

6. L. A. Fialkow, A note on quasisimilarity of operators, Acta Sci. Math. (Szeged) 39 (1977), 67-85.

7. C. Foias, C. Pearcy and D. Voiculescu, The staircase representation of biquasitriangular operators, Michigan Math. J. 22 (1976), 343-352.

8. T. B. Hoover, Quasisimilarity of operators, Illinois J. Math. 16 (1972), 678-686. 
9. A. Lambert, Strictly cyclic operator algebras, Ph.D. Thesis, University of Michigan, 1970.

10. M. Radjabalipour, On majorization and normality of operators, Proc. Amer. Math. Soc. 62 (1977), 105-110.

11. H. Radjavi and P. Rosenthal, On roots of normal operators, J. Math. Anal. Appl. 34 (1971), 653-664.

12. W. C. Ridge, Approximate point spectrum of a weighted shift, Trans. Amer. Math. Soc. 147 (1970), 349-356.

13. A. Shields, Weighted shift operators and analytic function theory, Topics in Operator Theory, Math. Surveys, no.13, Amer. Math. Soc., Providence, R.I., 1974, 49-128.

14. J. G. Stampfli and B. L. Wadhwa, An asymmetric Putnam-Fuglede theorem for dominant operators, Indiana Univ. Math. J. 26 (1976), 359-365.

Department of Mathematics, The University of Texas at Austin, Austin, TeXas 78712 